\title{
Effect of Antibiotic Prophylaxis on Serum CRP Level Immediately Following Periodontal Treatment: An Experimental Clinical Study
}

\author{
Basak Boduroglu ${ }^{1}$, Nilsun Bagis ${ }^{1}$, Cem A Gurgan ${ }^{2}$ and Hamit S Bostanci ${ }^{1}$ \\ ${ }^{1}$ Faculty of Dentistry, Department of Periodontology, Ankara University, Turkey \\ ${ }^{2}$ Faculty of Dentistry, Department of Periodontology, Erciyes University, Turkey
}

*Corresponding author: Assoc Prof Nilsun Bagis, Faculty of Dentistry, Department of Periodontology, Ankara University, Ankara, Turkey, Tel: 00-90-312-296-5679, Fax:00-90-312-212-3954,E-mail: nilsunbagis@yahoo.com

\begin{abstract}
Background: The immediate rise in serum C-reactive Protein (CRP) levels associated with the delivery of the subgingival instrumentation and the associated bacteremia and tissue damage was shown shortly after periodontal treatment was delivered. However, additive effect of the protection against bacteremia on immediate alterations in CRP levels has not been evaluated. Aim of this study was to determine the immediate effect of single session of non-surgical periodontal treatment with or without antibiotic prophylaxis on serum levels of CRP.

Methods: Forty systemically healthy subjects with chronic periodontitis were randomly assigned two groups. Clinical periodontal parameters and smoking status were recorded. Serum CRP levels were determined using nephelometric method at baseline and 1, 3 and 7 days after treatment.

Results: Except for the difference between baseline and day 7 , there were significant differences $(p<0.001)$ in CRP levels between the evaluation days in both groups. However, the differences between the groups for the alterations were not significant.

Conclusions: In agreement with previous observations, periodontitis and traumatic effect of periodontal treatment seem to contribute to systemic inflammation detected by the presence of immediate rise in CRP level. Additional administration of prophylactic antibiotic did not alter this immediate systemic response.
\end{abstract}

\section{Keywords}

Antibiotic prophylaxis, CRP, Periodontal treatment

\section{Introduction}

Numerous small and large scale cross-sectional studies have shown a positive association between chron- ic periodontitis and elevated serum C-reactive Protein (CRP) levels [1]. However, in order to determine whether this increase was due to periodontal infections or not, the possible effects of periodontal therapy on CRP levels were also investigated in numerous studies [2,3-7]. Results of these clinical studies indicated that treatment of periodontal infections, whether by standard or intensive mechanical therapy, adjunctive topical antibiotic application, or extraction, could significantly lower serum levels of CRP in 2 to 6 months' period. It was also shown that mechanical trauma produced by non-surgical periodontal treatment has caused a dramatic rise in serum CRP levels, even at the first day following treatment $[4,7]$. Also, transient bacteremia has been well documented immediately following scaling and root planning with sensitive methods [8-10]. Lipopolysaccharide and other cell wall products from periodontal bacteria can indirectly stimulate the liver to produce CRP. In addition to this, presence of periodontitis dramatically increases the risk for endotoxins being transferred to the systemic circulation. Increased endotoxin levels in the vasculature can initiate the proinflammatory response. Two main processes: The bacteremia or lipopolysaccharide related responses; and the pro-inflammatory cytokines are responsible for the direct rise in CRP levels. The consequence of the trauma produced by mechanical treatment was shown by D'Aiuto, et al. who found sharp increases for pro-inflammatory cytokines (i.e.: IL-1b and TNFa) in addition to CRP level at day 1 and 7 following treatment [7]. However, the effect of bacteremia on CRP levels following mechanical periodontal treatment has not been evaluated. Since the bacteremia was considered as 
one of the mechanism for the alteration in CRP level, we designed an experimental clinical study in order to evaluate the immediate (one day after) and very short term (one week period) effect of single session of non-surgical periodontal treatment with or without antibiotic prophylaxis on the serum levels of CRP.

\section{Material and Methods}

\section{Study design and experimental population}

The study was a prospective single blind controlled intervention trial with 7-day follow-up. Approval for the study was obtained from the Ethical Committee of Ankara University Faculty of Dentistry (Ref. no: 002/34, Approval date: 18.09.2002) to recruit adult patients with chronic periodontitis who required treatment.

Patients with systemic diseases which are established as risk factors for both periodontal disease and systemic CRP elevation were not included in the study. Pregnant women and individuals with acute or chronic medical disorders, including cardiovascular disorders, diabetes mellitus, viral, fungal and bacterial infections, trauma or recent tooth extractions, patients who underwent periodontal therapy within the past 6 months and used antibiotic within the past 3 months, and had allergic reactions against penicillin were not included in the study. All the patients had at least 20 teeth and periodontal pockets $\geq 5 \mathrm{~mm}$, attachment loss, presence of bleeding on probing and radiographical evidence of alveolar bone loss for at least 5 teeth.

Sample size was not based on formal power calculations due to the absence of available data at the time the study commenced.

The study material consisted of 40 chronic periodontitis patients who accepted to participate to the study and gave their informed written consent. The mean age of the patients was $42.57 \pm 5.44$ y.o. (range: $29-56$ y.o.). There were 17 women ( $41.82 \pm 5.91$, range: $34-56$ y.o.) and 23 men (43.13 \pm 6.94 , range: $29-54$ y.o.).

\section{Study outline}

A baseline visit was conducted by a blind well trained clinician who collected a complete medical history, standard clinical periodontal parameters, and blood samples. A periodontal treatment phase was carried out by an experienced periodontist who was unaware of study protocol. The patients were re-examined 1,3 and 7 days after the completion of treatment when the blood samples were collected.

\section{Clinical parameters}

All study participants were evaluated clinically at their first visit by one trained and calibrated examiner (B.B.). Calibration was performed before the study with 5 volunteer subjects. Reproducibility was determined by calculation of the percentage of sites examined where the scores were repeated exactly or to an accuracy of $1 \mathrm{~mm}$. Assessment of the mean difference in the scores (with
85\% accuracy) between two examinations indicated there was no systematic bias in measurements. Number of teeth, Plaque Index (PI) [11], Gingival Index (GI) [12], Probing Pocket Depth (PPD), Clinical Attachment Level (CAL), Suppuration (S) and Bleeding on Probing (BOP) [13] were recorded. Pocket depth and CAL were measured using a Williams' probe ('O' Probe with Williams, University of Michigan) at six sites around each tooth (mesio-buccal, mid-buccal, disto-buccal, mesio-lingual, mid-lingual, and disto- lingual). The average scores for whole-mouth PI, GI, PPD, CAL and the number of sites with BOP were divided by the total number of sites per mouth and multiplied by 100 for each subject.

Demographic information, including age, smoking status, frequencies tooth brushing and interdental cleaning were obtained by interviews. The Body Mass Index (BMI) was defined as the weight in kilograms divided by the square of height in meters, measured in the clinic.

\section{Periodontal treatment}

Following the baseline evaluation, the patients were randomly assigned to one of the two groups by another clinician. The first group received only a single session Non-Surgical Periodontal Therapy (NSPT). The second group received a single dose of amoxicillin of $2 \mathrm{gm}$ one hour prior to Non-Surgical Periodontal Therapy (AB+NSPT) according to the latest recommendations of American Heart Association. The periodontal treatment in both groups was comprised of a single session non-surgical full-mouth scaling and root planning performed by using hand and ultrasonic instruments under local infiltrative anesthesia, where necessary. The time period of treatment sessions was 2-4 hours depending on the extent of periodontal problem.

\section{Blood collection and analysis}

For the determination of serum CRP levels, $5 \mathrm{ml}$ of blood samples were obtained from each patient. The blood samples were delivered to the laboratory within the same day. Conventional and for the values below $1 \mathrm{mg} / \mathrm{l}$ high-sensitivity CRP analysis were utilized by Laboratory of Immunology, the Ankara University Faculty of Medicine, using nephelometric method (Images, Beckman-Coulter, USA).

\section{Statistical analysis}

The obtained data were analyzed using SPSS for Windows 10.0 statistical analysis program. The intra-group evaluations between evaluation days were analyzed with Friedman test. The inter-group comparison of the differences between the evaluations days were analyzed with Mann Whitney $U$ test. The differences were considered significant at $p<0.05$.

\section{Results}

All the patients in both groups completed the post-treatment period without any complication. No adverse effects 
Table 1: Frequencies and mean \pm standard deviation of demographic and periodontal parameters.

\begin{tabular}{|c|c|c|c|}
\hline Parameters & Categories & NSPT $^{*}$ & $A B+N S P T$ \\
\hline Age & & $42.80 \pm 7.05$ & $42.35 \pm 6.03$ \\
\hline (Min.-max.) & & $(29-56)$ & $(31-53)$ \\
\hline Woman/Man & & $12 / 8$ & $5 / 15$ \\
\hline Smoking & Yes/No & $11 / 9$ & $14 / 6$ \\
\hline Tooth brushing & No & 5 & 9 \\
\hline \multirow[t]{2}{*}{ Frequency } & Once & 12 & 10 \\
\hline & Twice & 3 & 1 \\
\hline Inter-proximal cleaning & Yes/No & $6 / 14$ & $6 / 14$ \\
\hline Body Mass Index (BMI) & & $25.79 \pm 4.11$ & $24.47 \pm 3.12$ \\
\hline (Min.-max.) & & (19.62-34.29) & $(19.03-29.05)$ \\
\hline \multirow[t]{3}{*}{ BMI categories } & < 25 (normal weight) & 11 & 9 \\
\hline & $\geq 25$ - $\leq 30$ (overweight) & 6 & 11 \\
\hline & $>\quad 30$ (obese) & 3 & 0 \\
\hline Plaque index & & $1.70 \pm 0.37$ & $1.75 \pm 0.51$ \\
\hline (Min.-max.) & & $(0.98-2.60)$ & $(1.03-2.86)$ \\
\hline Gingival index & & $1.17 \pm 0.16$ & $1.10 \pm 0.17$ \\
\hline (Min.-max.) & & $(0.83-1.49)$ & $(0.79-1.49)$ \\
\hline Bleeding on probing & & $0.73 \pm 0.21$ & $0.83 \pm 0.16$ \\
\hline (Min.-max.) & & $(0.42-0.98)$ & $(0.50-1.00)$ \\
\hline Pocket depth & & $3.62 \pm 0.46$ & $3.73 \pm 0.46$ \\
\hline (Min.-max.) & & $(3.06-4.83)$ & $(3.05-4.73)$ \\
\hline Attachment level & & $4.24 \pm 0.57$ & $4.22 \pm 0.67$ \\
\hline (Min.-max.) & & $(3.46-5.41)$ & $(3.22-5.54)$ \\
\hline Suppuration & & $0.01 \pm 0.22$ & $0.02 \pm 0.05$ \\
\hline (Min.-max.) & & $(0-0.08)$ & $(0-0.22)$ \\
\hline
\end{tabular}

${ }^{*}$ No significant difference between groups.

Table 2: Mean \pm standard deviation of CRP levels (mg/L).

\begin{tabular}{|c|c|c|c|c|c|c|}
\hline CRP level & p value & NSPT $^{*}$ & p value & p value & $A B+N S P T$ & p value \\
\hline $\begin{array}{l}\text { Baseline } \\
\text { (Min.-max.) } \\
\text { Median }\end{array}$ & $<0.001$ & $\begin{array}{l}3.17 \pm 2.29 \\
(1.00-10.30) \\
2.58\end{array}$ & & $<0.001$ & $\begin{array}{l}3.67 \pm 4.21 \\
(1.00-20.10) \\
2.85\end{array}$ & \\
\hline $\begin{array}{l}\text { Day } 1 \\
\text { (Min.-max.) } \\
\text { Median }\end{array}$ & $<0.001$ & $\begin{array}{l}9.03 \pm 5.53 \\
(2.69-21.40) \\
7.21\end{array}$ & $<0.001$ & $<0.001$ & $\begin{array}{l}9.25 \pm 5.89 \\
(1.00-22.30) \\
9.80\end{array}$ & $<0.001$ \\
\hline $\begin{array}{l}\text { Day } 3 \\
\text { (Min.-max.) } \\
\text { Median }\end{array}$ & \begin{tabular}{c|}
\cline { 2 - 2 } \\
$<0.05$
\end{tabular} & $\begin{array}{l}5.88 \pm 3.99 \\
(0.46-16.40) \\
5.45\end{array}$ & & $<0.01$ & $\begin{array}{l}7.43 \pm 6.79 \\
(0.57-31.80) \\
6.50\end{array}$ & \\
\hline $\begin{array}{l}\text { Day } 7 \\
\text { (Min.-max.) } \\
\text { Median }\end{array}$ & & $\begin{array}{l}3.69 \pm 2.19 \\
(0.17-7.68) \\
3.91\end{array}$ & & & $\begin{array}{l}4.19 \pm 4.97 \\
(0.15-22) \\
3.32\end{array}$ & \\
\hline
\end{tabular}

*No significant difference between groups.

were reported during the 7-day evaluation period.

The frequencies of age, sex, smoking status, oral hygiene habits, BMI and descriptive values of CRP levels on the evaluation days and periodontal indices obtained at baseline for both groups are shown in Table 1 . There were no significant differences between the groups for all the demographic and periodontal parameters.

For the alterations of CRP levels among the evaluation days, there was nearly a 3-fold increase in both groups when baseline values were compared with day 1 values $(p<0.001)$. Except for the difference between baseline and day 7 , there were significant differences among evaluation days in both groups. However, the differences between the groups for CRP levels at all evaluation days were not at a significant level (Table 2).

The CRP values of each patient at baseline and each evaluation day for both groups are shown in Figure 1 . There were 3 patients (one in NSPT (i.e.: \#1) and 2 in AB+NSPT (i.e.: \#8 and 17 groups) who had greater CRP level on the following days compared to the level measured at day 1.

No significant difference was found between smokers and non-smokers in both groups, except for day 7. However, the difference for CRP levels of non-smokers between the NSPT $(4.10 \pm 2.06 \mathrm{mg} / \mathrm{L})$ and $\mathrm{AB}+\mathrm{NSPT}$ 

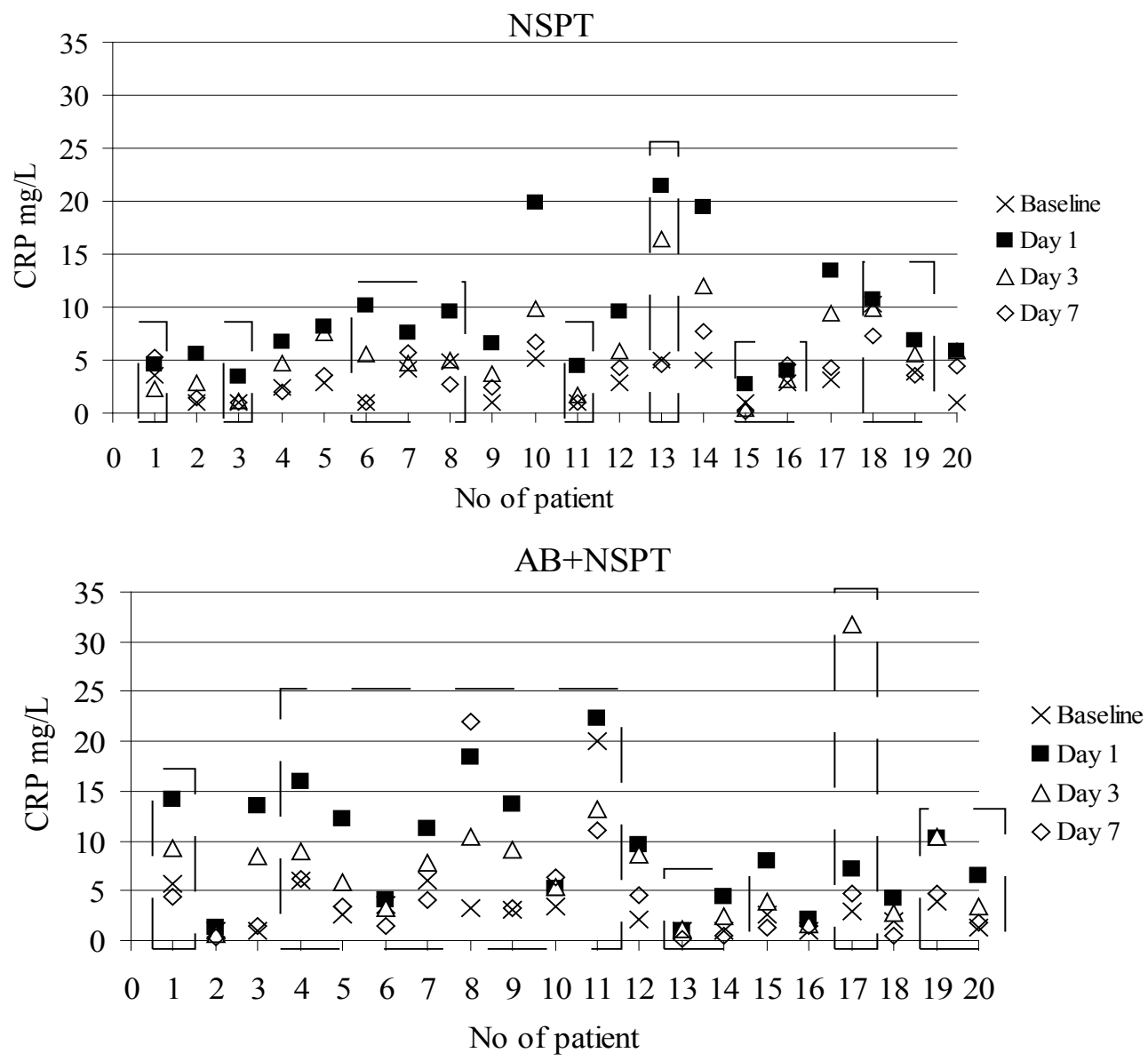

Figure 1: Individual CRP values.

*Dotted areas represent smoker patients.

Table 3: Mean \pm standard deviations of CRP levels (mg/L) according to smoking status of the patients in both groups.

\begin{tabular}{|c|c|c|c|c|c|c|}
\hline & & NSPT & & & $A B+N S P T$ & \\
\hline & Non-smoker (n:9) & p value ${ }^{*}$ & Smoker (n:11) & Non-smoker (n:6) & p value ${ }^{*}$ & Smoker (n:14) \\
\hline $\begin{array}{l}\text { CRP Baseline } \\
\text { (Min.-max.) } \\
\text { Median }\end{array}$ & $\begin{array}{l}2.73 \pm 1.59 \\
(1-5.18) \\
2.83\end{array}$ & N.S. & $\begin{array}{l}3.53 \pm 2.76 \\
(1-10.30) \\
3.64\end{array}$ & $\begin{array}{l}1.59 \pm 0.68 \\
(1-2.56) \\
1.45\end{array}$ & $<0.05$ & $\begin{array}{l}4.55 \pm 4.77 \\
(1-20.1) \\
3.31\end{array}$ \\
\hline $\begin{array}{l}\text { CRP Day } 1 \\
\text { (Min.-max.) } \\
\text { Median }\end{array}$ & $\begin{array}{l}10.58 \pm 5.67 \\
(5.54-19.90) \\
8.16\end{array}$ & N.S. & $\begin{array}{l}7.77 \pm 5.33 \\
(2.69-21.40) \\
6.84\end{array}$ & $\begin{array}{l}6.47 \pm 4.73 \\
(1.34-13.5) \\
6.08\end{array}$ & N.S. & $\begin{array}{l}10.44 \pm 6.09 \\
(1-22.3) \\
10.75\end{array}$ \\
\hline $\begin{array}{l}\text { CRP Day } 3 \\
\text { (Min.-max.) } \\
\text { Median }\end{array}$ & $\begin{array}{l}6.87 \pm 3.07 \\
(2.79-12) \\
5.80\end{array}$ & N.S. & $\begin{array}{l}5.08 \pm 4.60 \\
(0.46-16.40) \\
4.72\end{array}$ & $\begin{array}{l}4.33 \pm 4.73 \\
(0.57-8.7) \\
3.33\end{array}$ & N.S. & $\begin{array}{l}8.76 \pm 7.52 \\
(1.6-31.8) \\
8.39\end{array}$ \\
\hline $\begin{array}{l}\text { CRP Day } 7 \\
\text { (Min.-max.) } \\
\text { Median }\end{array}$ & $\begin{array}{l}4.10 \pm 2.06 \\
(1.46-7.08) \\
4.23\end{array}$ & N.S. & $\begin{array}{l}3.35 \pm 2.33 \\
(0.17-7.23) \\
3.57\end{array}$ & $\begin{array}{l}1.61 \pm 1.52 \\
(0.31-4.56) \\
1.38\end{array}$ & $<0.05$ & $\begin{array}{l}5.29 \pm 5.55 \\
(0.15-22) \\
4.27\end{array}$ \\
\hline$p$ value ${ }^{* *}$ & \llcorner & & $<0.05$ & 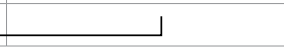 & & \\
\hline
\end{tabular}

*Difference between non-smoker and smoker; ${ }^{* *}$ Difference between treatment groups; N.S: Not Significant.

$(1.61 \pm 1.52 \mathrm{mg} / \mathrm{L})$ groups was at a significant level $(\mathrm{p}<$ $0.05)$. In AB+NSPT group, the difference between smokers $(4.55 \pm 4.77 \mathrm{mg} / \mathrm{L})$ and non-smokers $(1.59 \pm 0.68$ $\mathrm{mg} / \mathrm{L})$ at baseline $(\mathrm{p}<0.05)$ and day $7(5.29 \pm 5.55 \mathrm{mg} / \mathrm{L}$ for smokers vs. $1.61 \pm 1.52 \mathrm{mg} / \mathrm{L}$ for non-smokers, $p<$ 0.05 ) were also at a significant level (Table 3 ).

\section{Discussion}

Generally, in the clinical practice non-surgical periodontal therapy comprising scaling and root planning is completed in multiple sessions depending on the teeth involved. Knowing the pro-atherogenic features of CRP which can be influenced by bacteremia and/or inflammation, some protective measures must be taken in order to prevent possible harm to the patient during routine periodontal therapy.

Bacteremia frequently occurs after dental treatment, the magnitude of the episodes usually being within a range of a few CFU/ml [14]. As a result of bacteremia, which was detected in $13-70 \%$ of periodontitis patients after scaling, cytokine synthesis by stimulated 
blood leukocytes was likely to occur $[9,14]$. As bacteremia and tissue damage are produced, local and systemic production of pro-inflammatory cytokines has been described as a component of host defense $[15,16]$. In order to modulate this process, anti-inflammatory molecules (such as IL-1Ra, IL-6) appear on the scene to control inflammation and stimulate the hepatic synthesis of acute-phase proteins (e.g.; CRP) [17].

Ide, et al. [18] evaluated the rapid changes in CRP levels during non-surgical periodontal treatment in 23 non-smoking adults with chronic periodontitis. Although significant increases were observed in circulating levels of TNFa and IL-6, they found no difference in CRP levels between before and 120 minutes after treatment.

In our study, a prophylactic dosage of systemic antibiotic (i.e. $2 \mathrm{~g}$ amoxicilin 1 hour before treatment) was prescribed as in the test group. The reason of choosing prophylactic dosage was that $2 \mathrm{gm}$ of amoxicilin was one of the highest single doses that could be safely administered especially, when a single and intensive session of treatment was scheduled. Antibiotics may prevent endocarditis either by killing bacteria or by damaging them to an extent that the host defense can then destroy them. Therefore, the antibiotic may work before the bacteria enter the bloodstream, after they enter the bloodstream or on colonies of bacteria. The primary mechanism by which antibiotic prophylaxis could occur has not been established but a number of studies showed that bacteremia was reduced both in quantity and time in the presence of antibiotics [19]. Amoxicillin is bactericidal that is useful in treating the acute phase of odontogenic infection, in addition to prevention of associated complications. Due to its effectiveness against facultative aerobic and anaerobic pathogens, it is considered to be the antibiotic of choice in the treatment of infections of mixed etiology in the oral cavity [20].

CRP levels in our study increased nearly 3-fold after one day and returned almost to baseline values just 7 days after treatment (Table 2). Its concentration changes followed the pattern of a classic acute-phase marker with a rise within 24 hours. Bulut, et al. [21] have assessed the value of routine antibiotic prophylaxis in comparison with placebo administration, in impacted mandibular third molar surgery using CRP levels measured prior to surgery $(5 \mathrm{mg} / \mathrm{L})$ and on post-operative days 1, 3 and 7 . According to their results, no significant difference between the antibiotic prophylaxis and placebo groups with regard to change in CRP level was observed. However, there was also a significant rise in CRP levels at day 1 (range: $12.3-13.1 \mathrm{mg} / \mathrm{L}$ ) and then progressive decline to pre-operative level at day 7 (range: $5.3-5.4 \mathrm{mg} / \mathrm{L}$ ), in both groups. Both our and the study done by Bulut, et al. [21] have failed to show any effect of antibiotic prophylaxis on immediate rise in CRP levels following extraction of impacted third molars or intensive non-surgical periodontal treatment. Therefore, the immediate elevations of CRP levels could be attributed to mechanical manipulation-induced aseptic traumatic inflammation.

D'Aiuto, et al. performed 2 studies including 14 [4] and 55 [7] otherwise healthy subjects suffering from severe chronic periodontitis and evaluated the immediate effect of intensive non-surgical periodontal treatment completed within 6 hours, on CRP levels obtained 1, 3, 5 and 7 days after treatment. According to their results, intensive periodontal treatment produced an acute systemic inflammatory response of 1 week duration with a 5 to 10 -fold increase in CRP $(p<0.001)$ detected on first post-operative day (range: $12.49-17.5 \mathrm{mg} / \mathrm{L}$ ) compared to baseline (range: $1.61-2.5 \mathrm{mg} / \mathrm{L}$ ). They also observed higher CRP levels compared to pre-treatment period during the consecutive post-operative days (i.e.: day 3 , 5 and 7). Although our baseline values (range: 3.17-3.67 $\mathrm{mg} / \mathrm{L}$ ) were higher than their values, the similar pattern of alteration that we observed in our both groups were in concordance with their findings (Table 3).

In a previous study performed in our clinic [22], subjective and objective reactions in a group of chronic periodontitis patients following full-mouth daily scaling and root planning for 4 consecutive days compared to a group treated once a week for 4 weeks were evaluated. There were significant differences between the study groups for lymphadenopathy and fatigue symptoms. The percent value of lymphadenopathy-positive patients in daily treated group was twice of that weekly treated ones. Mongardini, et al. [23] reported that half of the patients who underwent intensive subgingival instrumentation over a short period reported pyrexia over the following 12-24 hours as anecdotal evidence. Those reported changes in the above mentioned two studies could be attributed to systemic elevation of pro-inflammatory cytokines and acute-phase proteins.

The classical definition of inflammation has been "the response to tissue injury". Injury, resulting in distressed cells, is the trigger, and inflammation the consequence. The tissue injury that occurs when cells have been stressed by an insult of some kind often results in metabolic impairment and local expression of pro-inflammatory cytokines. Tissue injury does not necessarily imply cell death. There is a spectrum of responses to insult, ranging from sub-inflammatory, non-necrotic changes in response to mild stresses to full blown overt inflammation in response to major injury [22].

Although it has generally been assumed that a CRP response indicates an underlying inflammatory process, it is equally likely that it reflects distressed or injured cells, as in the case of traumatic effect of periodontal treatment and the following healing response.

The acute phase response is a continuum and daily life is full of minor degrees of tissue injury and environmental irritants. CRP has been shown to play a role in the 
pathogenesis of atherosclerosis through different mechanisms including binding the phosphocholine of oxidized low-density lipoproteins, upregulating the expression of adhesion molecules in endothelial cells, increasing low-density lipoprotein uptake into macrophages, inhibiting endothelial nitric-oxide synthase expression in aortic endothelial cells, and increasing plasminogen activator inhibitor-1 expression and activity [24]. Levels of CRP less than $1 \mathrm{mg} / \mathrm{L}$ are desirable and reflect a low overall cardiovascular risk. CRP levels between 1 and 3 $\mathrm{mg} / \mathrm{L}$ are indicative of moderate risk, while levels of CRP in excess of $3 \mathrm{mg} / \mathrm{L}$ suggest quite elevated vascular risk [25]. However, CRP levels over $10 \mathrm{mg} / \mathrm{L}$ reflect clinically significant inflammatory states, "macro-inflammation", as long recognized. Although intermediate values may indicate the presence of a broad array of minor inflammatory states, they may also reflect genetic factors, demographic variables, behavioral and dietary patterns which, although not overtly inflammatory, may represent minor degrees of tissue injury [26]. This condition was also observed in our both groups with the presence of inter-individual variations in magnitude and duration of acute phase response (Figure 1).

Approximately, sixty-three percent of our whole study population (55\% of NSPT and $70 \%$ of $A B+N S P T)$ was smoker. Although not at a significant level, except for day 7, there were higher CRP values for non-smokers in NSPT group. The difference between the groups for non-smoker patients at day 7 was more than 2-times higher in the favor of AB+NSPT group $(p<0.05)$. Caution is needed, however, in interpreting these results given small number of patients (9 vs. 6) included. In $A B+N S P T$ group higher and significant values of CRP levels were observed both at baseline $(p<0.05)$ and day 7 $(p<0.05)$ for smokers compared to non-smokers (Table 3). These results for smoker patients were in concert with D'Aiuto, et al. [6] According to their results, smokers showed less decrease in CRP levels in respond to standard non-surgical periodontal treatment. Opposite to our results, smokers could have only responded to intensive treatment where they have administered locally applied minocycline as an adjunct to periodontal treatment in their study.

The present study was designed only to evaluate the immediate alterations in CRP levels. Therefore, there are a series of limitations to our investigation that we ought to report. Firstly, the serum/plasma quantification of bacteremia by using microbiological tests before and after treatment was not evaluated. However, the recommendations of AHA against bacteremia were followed. Secondly, although very short-term effect was evaluated, the mean CRP levels at the end of evaluation period were almost similar to baseline values in both groups. Effect of periodontal treatment on CRP levels in longer periods, as mentioned elsewhere [1] was not evaluated. A third limitation of this study is the number of individuals included in this analysis is relatively small and these findings should be interpreted.

In agreement with previous observations [4,7], within the limits of our study, analysis of our data indicated that periodontitis and traumatic effect of periodontal treatment seem to contribute to systemic inflammation. However, the local inflammatory healing response following non-surgical periodontal therapy was seemed to have more contribution to the dramatic and immediate raise in CRP levels than bacteremia. Given the evidence of significant entrance of bacteria into the systemic circulation following periodontal instrumentation $[9,10]$, our data describe the systemic inflammatory changes occurring after periodontal treatment even though the presence of systemic high dosage of antibiotic. This pattern of alterations should be considered when patients with systemic diseases (e.g.: cardiovascular problems, diabetes mellitus and rheumatoid arthritis) are being treated especially, multiple non-surgical treatment sessions are being planned.

As a suggestion for future studies, systemic and local antibiotic administrations and anti-inflammatory therapies (such as COX-2 inhibitors, lipoxins, chemically modified tetracycline and subantimicrobial dose of doxycycline) [27] as adjunct to periodontal therapy should be considered in order to evaluate the possible contribution of periodontal inflammation and treatment either as a part of healing response or against infection, to the systemic changes.

\section{Acknowledgment}

This work was supported by a grant from Ankara University Research Foundation (No. 20030802050).

\section{Conflict of Interest}

The authors declare that they have no conflict of interests.

\section{References}

1. Ioannidou E, Malekzadeh T, Dongari-Bagtzoglou A (2006) Effect of Periodontal Treatment on Serum C-Reactive Protein Levels: A Systematic Review and Meta-Analysis. J Periodontol 77: 1635-1642.

2. Mattila $\mathrm{K}$, Vesanen $\mathrm{M}$, Valtonen $\mathrm{V}$, Nieminen $\mathrm{M}$, Palosuo $\mathrm{T}$, et al. (2002) Effect of treating periodontitis on C-reactive protein levels: a pilot study. BMC Infect Dis 2: 30-32.

3. Ide M, McPartlin D, Coward PY, Crook M, Lumb P, et al. (2003) Effect of treatment of chronic periodontitis on levels of serum markers of acute-phase inflammatory and vascular responses. J Clin Periodontol 30: 334-340.

4. D'Aiuto F, Nibali L, Mohamed-Ali V, Vallance P, Tonetti MS (2004) Periodontal therapy: a novel non-drug-induced experimental model to study human inflammation. J Periodont Res 39: 294-299.

5. D'Aiuto F, Parkar M, Andreaou G, Suvan J, Brett PM, et al. (2004) Periodontitis and systemic inflammation: Control of the local infection is associated with a reduction in serum inflammatory markers. J Dent Res 83: 156-160.

6. D'Aiuto F, Nibali L, Parkar M, Suvan J, Tonetti MS (2005) Short-term effects of intensive periodontal therapy on se- 
rum inflammatory markers and cholesterol. J Dent Res 84: 269-273.

7. D'Aiuto F, Parkar M, Tonetti MS (2005) Periodontal therapy: a novel acute inflammatory model. Inflamm Res 54: 412-414.

8. D'Aiuto F, Parkar M, Nibali L, Suvan J, Lessem J, et al. (2006) Periodontal infections cause changes in traditional and novel cardiovascular risk factors: results from a randomized controlled clinical trial. Am Heart J 151: 977-984.

9. Kinane DF, Riggio MP, Walker KF, MacKenzie D, Shearer B (2005) Bacteraemia following periodontal procedures. J Clin Periodontol 32: 708-713.

10. Forner L, Larsen T, Kilian M, Holmstrup P (2006) Incidence of bacteremia after chewing, tooth brushing and scaling in individuals with periodontal inflammation. J Clin Periodontol 33: 401-407.

11. Silness J, Loe H (1966) Periodontal disease in pregnancy. III. Response to local treatment. Acta Odontol Scand 24: 747-759.

12. Loe H, Silness J (1963) Perıodontal disease in pregnancy. I. Prevalence and severity. Acta Odontol Scand 21: 533551.

13. Muhleman HR, Son S (1971) Gingival sulcus bleeding: a lading symptom in initial gingivitis. Helv Odontol Acta 15: 107-113.

14. Heimdahl A, Hall G, Hedberg M, Sandberg H, Söder PO, et al. (1990) Detection and quantitation by lysis filtration of bacteremia after different oral surgical procedures. J Clin Microbiol 28: 2205-2209.

15. Birkedal-Hansen H (1993) Role of cytokines and inflammatory mediators in tissue destruction. J Periodontal Res 28: 500-510.

16. Madianos PN, Bobetsis YA, Kinane DF (2005) Generation of inflammatory stimuli: how bacteria set up inflammatory responses in the gingiva. J Clin Periodontol 32: 57-71.

17. Gabay C, Kushner I (1999) Acute-phase proteins and oth- er systemic responses to inflammation. N Engl J Med 340: 448-454.

18. Ide M, Jagdev D, Coward PY, Crook M, Barclay GR, et al. (2004) The short-term effects of treatment of chronic periodontitis on circulating levels of endotoxin, C-reactive protein, tumor necrosis factor-alpha, and interleukin-6. J Periodontol 75: 420-428.

19. Singh J, Straznicky I, Avent M, Goss AN (2005) Antibiotic prophylaxis for endocarditis: time to reconsider. Aus Dent $J$ Medications 50: 60-68.

20. Kuriyama T, Karasawa T, Nakagawa K, Saiki Y, Yamamoto $\mathrm{E}$, et al. (2000) Bacteriologic features and antimicrobial susceptibiliry in isolates from orofacial odontogenic infections. Oral Surg Oral Med Oral Pathol Oral Radiol Endod 90: 600-608.

21. Bulut E, Bulut S, Etikan I, Koseoglu O (2001) The value of routine antibiotic prophylaxis in mandibular third molar surgery: acute-phase protein levels as indicators of infection. J Oral Science 43: 117-122.

22. Eren KS, Gürgan CA, Bostanci HS (2002) Evaluation of non-surgical periodontal treatment using 2 time intervals. J Periodontol 73: 1015-1019.

23. Mongardini C, van Steenberghe D, Dekeyser C, Quirynen M (1999) One stage full- versus partial-mouth disinfection in the treatment of chronic adult or generalized early-onset periodontitis. I. Long-term clinical observations. J Periodontol 70: 632-645.

24. Black S, Kushner I, Samols D (2004) C-reactive Protein. J Biol Chem 279: 48487-48490.

25. Ridker PM (2003) Cardiology Patient Page. C-reactive protein: a simple test to help predict risk of heart attack and stroke. Circulation 108: e81-e85.

26. Kushner I, Rzewnicki D, Samols D (2006) What does minor elevation of C-reactive protein signify? Am J Med 119: 166.

27. Salvi GE, Lang NP (2005) Host response modulation in the management of periodontal diseases. J Clin Periodontol 32: $108-129$ 